\title{
A study on the System Architecture and Key Technologies of Telematics Based on 3G
}

\author{
Lifang Tang \\ Department of Computer Engineering \\ Cangzhou Normal College \\ Cangzhou, Hebei Province, China \\ Tiandoudou2008@126.com
}

\author{
Chuanjin Wang \\ Department of Fine Arts \\ Cangzhou Normal College \\ Cangzhou, Hebei Province, China \\ 331757411@qq.com
}

\begin{abstract}
Telematics, the application of Internet of Things technology in the intelligent transportation system, has attracted keen attention of research mechanism at home and abroad. By introducing the basic conception of Telematics, the paper makes a summary and research on the technologies adopted in the system architecture of Telematics and put an emphasis on the solution of some key technologies.
\end{abstract}

Keywords- Telematics, system architecture, Key technology, intelligent transportation system

\section{INTRODUCTION}

The frequent traffic accidents are the nightmares for everyone, and the car enterprises and the government have always endeavored to reduce the traffic accidents. However, it is impossible to reach this goal overnight. Telematics provides a foundation to eliminate the traffic accidents. In the era of Telematics, intelligent vehicles can foresee the potential collision through interior sensors and take emergency action automatically to prevent the occurrence of the accidents jointly through the communication between the driver and the vehicle as well as the communication between vehicles.

\section{THE DEFINITION OF TELEMATICS}

Telematics is to set up electronic label on the vehicles, which can realize the extraction and effective utilization of attribute information on all vehicles on the internet information platform through Smart ID, carry out effective supervision on the operation status of the vehicle in accordance with varies requirements on the functions and provide integrated services.[1]

Intelligent Transport System is a real-time, accurate and efficient transportation integrated management and control system, which is established when advanced technology such as sensor technology, communications technology, data processing technology, network technology, automatic control technology, information publishing technology is applied to the whole transportation management system organically. Industry chains of IOV cover fields such as auto parts manufacturers, chip manufacturers, software providers, solution providers, network suppliers and so on.

Car node and information source obtained by means of wireless communication technologies such as the car itself and the car external attributes and use them effectively, so as to achieve the interoperability of the "human - vehicle - road - environment". [2]As the Internet allows people to achieve a "peer-to-peer information exchange," car networking "also make cars and trucks" dialogue ". Car network includes two parts: automotive electronics, and urban intelligent transportation. With increasingly intelligent cars and roads, more and more cars and side infrastructure equipped with communications equipment. Intelligent transportation can improve the efficiency of road use, a decrease of approximately $60 \%$ of the traffic jams, the short-distance transport efficiency by nearly $70 \%$, increased by two to three times the capacity of existing roads. Vehicles traveling within the intelligent transportation system, the number of stops by $30 \%, 13 \%$ to $45 \%$ reduction in travel time, vehicle use efficiency can be increased by $50 \%$ or more.[4]

\section{THE ARChitecture OF TElEMATICS}

As shown in Picture 1, Telematics can be divided into three layers. The first one is the sensing layer including the sensor systems like RFID, which is also the simplest layer. The second one is the interconnection layer. After sensing, the vehicles, the vehicle and the road should interconnect with each other. The third one is the cloud layer which can regulate and manage the vehicles by intelligent computing such as cloud computing.[3]

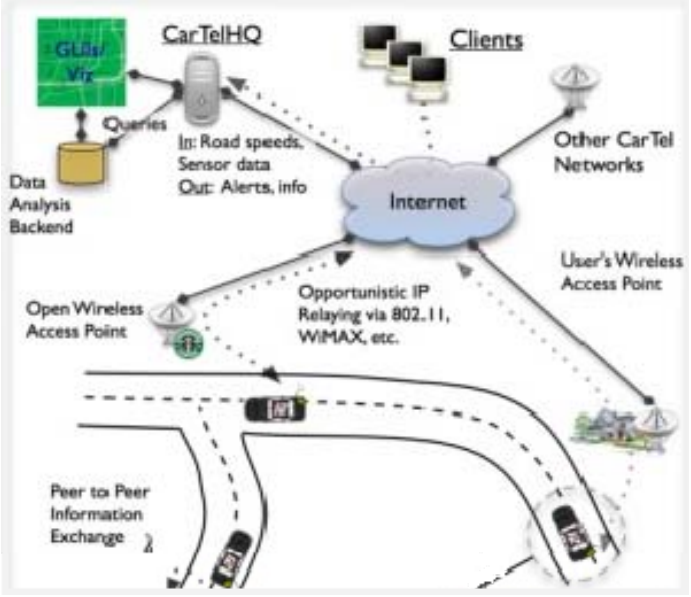

Figure 1. the Architecture of Telematics. 


\section{A. the Sensing Layer}

The sensing layer can be divided into two sub-layers. The lower layer mainly functions to identify the nodes of the network, sense the vehicles position, the driving speed, the traffic conditions and weather conditions and collect related data. The upper layer mainly functions to transmit data in self-organized networks(among the active CPS nodes). The physical devices that the sensing layer needs mainly include RFID label, reader-writer, various sensors (sense the information such as temperature, speed, and vehicle conditions), GPS, and camera. On this layer, RFID technology can be applied to transmit data.

\section{B. the Interconnection Layer}

The major function of the interconnection layer is to connect the Internet, finish the analysis processing of the data and transmit them in a far distance. Meanwhile, the network layer can be used to remotely monitor and manage the nodes in Telematics. The devices that this layer mainly utilizes are CPS nodes on the Internet, which acts as the router in the traditional network. It's natural that the traditional router isn't equipped with the control function that the Internet CPS nodes have. It should employ protocol conversion to connect Telematics and the Internet, which is because the protocol used by the lower layer of Telematics is different from TCP/IP. [5]

\section{C. the Cloud Layer}

Cloud computing is used to carry on comprehensive processing and analysis on the data of the obtained objects and provide various integrated services. Telematics system can acquire the service provided by the cloud computing according to demands and in a manner that is easy to expand through the network.

The cloud layer of Telematics can be further divided into two sub-layers. the lower layer is the application layer, which is primarily used to process the data. The specific services that Telematics provides can also be defined and realized on this layer. At present, it is well acknowledged that the middleware technology is a better choice for various services provided by Telematics. The upper layer is User Interface, which can define the ways and contents of user interaction. The devices on the application layer mainly comprise servers that provide network service and on-board computer which the clients use.

\section{The Key Technology of Telematics}

\section{A. $3 G$}

The principal character of $3 G$, the third generation mobile communication technology, is to provide a variety of mobile multimedia services, which can support a transmission rate of $144 \mathrm{~kb} / \mathrm{s}$ in high-speed mobile environment, of $384 \mathrm{kbs}$ in slow-speed mobile environment and of $2 \mathrm{Mb} / \mathrm{s}$ in the static condition. Its designing objective is proposed to provide better communication quality with higher system capacity than the Second Generation System. Otherwise, the utilization of seamless switching technology in the heterogeneous network can offer services for the end consumers by connecting the wireless network and Internet.

With the characteristics of high system capacity, sounder system communication quality and high bandwidth efficiency, 3G can generally meet the requirements on the transmission of Telematics application data. Telematic system of $3 \mathrm{G}$ communication technology is mainly consisted by $3 G$ data communication link (TD-SCDMA or CDMA 2000), monitoring center and several remotes. Adopting standard TCP/IP protocol, 3G data communication link can be directly operated on the interior wireless local area network of the communication departments. The visual signal sent by the front camera can be transmitted to the partial nodes with the help of the network video server through the network, which can directly transmit on the Internet on the partial nodes or connect the matrix if there is a matrix in the partial nodes and then transmit on the Internet. The monitoring center can constitute multilevel monitoring system through multilevel cascades, which can configure different peripheral hardwares based on the users' demands and the real situation. The peripheral hardwares for the application system of the remote is consisted by the following devices, the monitor server, $3 G$ data transmission module, warning collector, temperature and humidity sensor, video splitter, video camera, digital decoder, high-speed pan and zoom lens, microphone and loudspeaker. It can carry on real time monitoring on the pictures needed by the monitoring center, intelligent control on the traffic signal on the spot and warning cascade. The peripheral devices for the application system of the monitoring center contain the master control, 3G wireless router, monitoring array, video switch matrix, principal switch, video server and TV-wall, which can be configured flexibly according to the users' requirements.

\section{B. RFID}

RFID technology is touchless automatic identification technology on the basis of radio frequency theory, which can automatically identify the object by using the space coupling transmission attribute of the wireless radio frequency signal. As it is indicated in Picture 2, RFID system is usually consisted by the label, the reader, the antenna and the middleware.

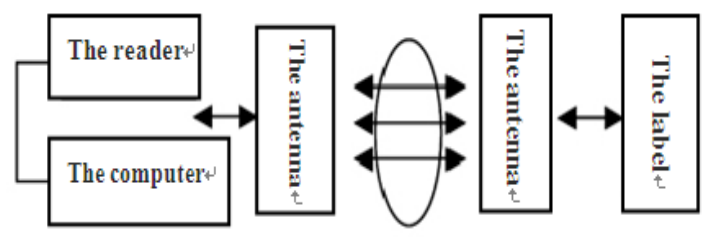

Figure 2. the construction of RFID system

The label: RFID contains coupling elements and chips. Every label has a unique electronic code which is attached on the objects to identify the target object and commonly known as electronic label or intelligent label. 
The reader/writer: this device is used to read the label information and can be designed in handheld type or fixed type.

Antenna: this device can transmit radio frequency signal between the RFID label and the reader.

The working principal of RFID: when the label enters the magnetic field, it can receive the radio frequency signal sent by the reader and send the product information stored in the chips depending on the energy acquired by induction current(passive tags) or take the initiative to send signals at a frequency(Tag, active tag). After the reader read and encode the information, it will deliver the encoded information to the center information system to carry on data processing.

Comparing with the traditional bar code technology, RFID technology has obvious advantage on the identification speed, identification distance, storage capacity, reading and writing ability and the environmental adaptation. Therefore, it has been applied in the social, economic and defense fields gradually.

The core of the idea to adopt RFID technology in the transportation information system is to install RFID readers on the both sides or the top of the road and to attach RFID label on every vehicle. The ID number of RFID card on every vehicle can be used as the key words to establish database and connecting the ID number of the label and the vehicle number can establish a parameters database for all vehicles. A FRID reader can connect many sets of antennas and identify the RFID read by a certain antenna logically. A reader with many sets of antennas can meet the need to monitor the vehicle movement condition on every traffic lane. RFID system has the ability to reliably identify every vehicle wherever it is, no matter whether it stops or moves, and no matter what whether it is (the image license plate identification method used before is easily affected by heavy rain, thick flog or stained license plate). Every electronic license plate has the unique encryption ID number and the reader can read several ID numbers at the same time when the speed is $300 \mathrm{~km}$ per hour and the distance is within 100 meters.

In addition, RFID can be used to collect the data correlation and precisely detect the group velocity of the vehicle flow, which is of great importance as it can directly reflect the condition of traffic congestion. The unique characteristic of vehicle ID number can be used to easily measure the time lag that the vehicle drives from a RFID detection point to another one and call the range difference between the two detection points (the geographic location of the two detection points is determined so the distance can be known), so that it can determine the group velocity that represents the movement of vehicle flow in this section. Moreover, by colleting and smoothing the information on some electronic labels on the nearby sections, it can describe the traffic condition on these sections more precisely and detailedly and release the traffic guidance information more accurately and timely.

The utilization of RFID can realize non-stopping toll. When the vehicle drives to the freeway entrance, the RFID reader will record the information data of the toll station entrance on the electronic labels. When this vehicle drives to
ETC channel exit for the next toll station, RFID will read the information on the labels and deliver it to backstage management system. After verified by ETC backstage charge management, the automatical charge can be completed through online bank at one time. Then turn on the green light or other go-ahead signals to control the lift rod of the barrier and direct the vehicle to pass normally. However, if it is verified that the vehicle cannot be allowed to pass legitimately, the red light or other stopping signals will maintain and direct the vehicle cannot pass normally. Meanwhile, the installed high-speed camera can record the related information data rapidly and process it by managerial personnel.

\section{NFC}

NFC, Near Field Communication, is a kind of wireless technology initiated by Philips and promoted under the joint efforts of some famous companies like Nokia and Sony. NFC is evolved from touchless RFID and interconnection technology, which integrates induction card reader, induction card and PTP (point to point), and can identify and change data with compatible devices in a short distance.

NFC is a complementary technology in the automotive field and can co-exist with the technologies that are more popular and mature in recent years, such as Bluetooth and Wi-Fi. The major advantage of NFC is the interior security. As the communication distance of this technology is restricted and the single PTP (point to point) connection also restricts the possibility to be intercepted, so it is relatively safer. [6]Furthermore, NFC technology support advanced encryption, which makes the system based on NFC suitable for the secure financial transaction and other types of secure transactions. NFC technology dramatically strengthens the intelligent performance of the interior devices and ensures that the data are safer and more convenient.

\section{Cloud Computing}

Cloud computing is a model that can obtain computing resources (network, server, storage, application and service) in a convenient way on demand though the Internet. These resources are from a sharing and configurable resource pool and can be acquired and released quickly.

Generally speaking, cloud computing has the following five characteristics: self service on demand, broad network access, resource pool, rapid elasticity and charge based on actual usage.

The cloud computing for Telematics is a mechanism with integrated planning, unified organization and unified allocation of resources based on centralized sharing of cloud computing resources, traffic resources and vehicle resources, which can optimize the whole traffic system to establish an integrated traffic system to meet various demands on transportation. It can not only cut down the total cost, but also change the ideology of constructing traffic system from "service decides the information" to "innovate service based on information integration". The openness of Telematics enables more users to participate in this system so as to broaden the information resource. Richer information will bring about a wider variety of application, which can attract 
much more users. If this virtuous cycle can keep moving, Telematics can become an open system.

\section{E. Intelligent Analysis}

Intelligent analysis technology is a kind of pattern recognition technology. According to a higher point of view, it belongs to the scientific scope of "Artificial Intelligence". That is to say the computer has some intelligent capability and can understand what have happened for a certain degree, rather than a series of sequence sets. In the field of traffic video monitoring, the information on license numbers is extracted and identified through continuous analysis on the video uploaded by the camera. It is quick to know whether there is someone who violates the regulation and breaks into a safe area or there happened an accident on this road" from a video image, so that it can fulfill the function of automatic detection and warning.

\section{CONCLUSION}

It is a new way for the intelligent traffic control system to organize a network by using $3 \mathrm{G}$ wireless networks to transmit the video of signal location on every traffic intersection and control information. In the near future, the vehicle can not only drive automatically, but also form a network connecting vehicles and vehicle and road to keep on communicating from time to time. In busy cities, under the control of intelligent traffic network, vehicles can shuttle rapidly and orderly. Cars will no longer drink oil, green charge station will flourish on the streets and people can charge their beloved cars at any time. This rosy imagination on the future automotive society will be realized with the application of Telematics in the field of commercial vehicles.

\section{REFERENCES}

[1] Wang Jianqiang, Wu Chenwen, "A Novel Opportunistic Rou-ting Protocol Applied Tto Vehicular Ad Hoc Networks,"The 5th International Conference On Computer Science \& Edu-cation. pp. 1005-1009,2010 (references)

[2] Hadaller D, Keshav S, Brecht T, et al. "Vehicular Opportun-istic Communication Under the Microscope," Proceeding of the 5th International Conference On Mobile Systems, Appli-cations And Services. USA: [s. n. ], 2007, USA. (references)

[3] Akyildiz I F, Wang Xudong, Wang Weilin, "Wireless Mesh Networks A Survey,” Computer Networks, pp. 445-487, April 2005. (references)

[4] Wang Jianqiang, Wu Chen Wen, Li Xiaojun, "The Car Networking Architecture A7nd Key Technology Research,” Microcomputer, pp. 156-158 ,April 2011. (references)

[5] Kevin C.Lee,Uichin Lee,Mario Gerla,et al.Geo-Opportunistic Routing for Vehicular Networks,J.IEEE Communications Magazine, pp. 164-170,May 2010(references)

[6] Hannes Hartenstein,Kenneth P.Laberteaux,et al. "A Tutorial Survey on Vehicular Ad Hoc Networks,”IEEE Communications Magazine, pp. 104-107,June 2008.(references) 